\title{
Relationships between Verbal and Visuospatial Working Memories and Source Monitoring among Young Children
}

\author{
Ikumi Ozawa, Masamichi Yuzawa \\ Graduate School of Education, Hiroshima University, Higashi-Hiroshima, Japan \\ Email: ozawan193@gmail.com
}

How to cite this paper: Ozawa, I., \& Yuzawa, M. (2018). Relationships between Verbal and Visuospatial Working Memories and Source Monitoring among Young Children. Psychology, 9, 945-957.

https://doi.org/10.4236/psych.2018.95059

Received: March 8, 2018

Accepted: May 7, 2018

Published: May 10, 2018

Copyright $(0) 2018$ by authors and Scientific Research Publishing Inc. This work is licensed under the Creative Commons Attribution International License (CC BY 4.0).

http://creativecommons.org/licenses/by/4.0/

\begin{abstract}
The results of previous studies examining the relationship between source monitoring and working memory in young children are not consistent. One of the reasons for this inconsistency is concerning the modalities of information with which working memory tasks deal. The present study investigated how young children's verbal and visuospatial working memory capacity would be related to respective source-monitoring tasks with visually or verbally presented stimuli. Children aged four $(n=21)$ and five years $(n=21)$ participated in this study. They completed two verbal working memory tasks, namely, the backward digit span and listening span tests; and two visuospatial working memory tasks, namely, a comparative line test and a rotated figure test. In the source-monitoring task, first, an adult man and an adult woman read two different picture books to groups of children. Subsequently, they read short sentences aloud and showed pictures to the children. Each child then performed recognition tests for the sentences and the pictures, and required to decide on the appropriate source. Hierarchical multiple regression analyses revealed the following results: The verbal working memory task (i.e., the backward digit span) was a significant factor explaining performance on the verbal source-monitoring task, whereas the visuospatial working memory task (i.e., a rotated figure test) explained the performance on the visual source-monitoring task.
\end{abstract}

\section{Keywords}

Source Monitoring, Verbal Working Memory, Visuospatial Working Memory, Young Children, Eyewitness Testimony

\section{Introduction}

Source monitoring refers to making an attribution about the origin of a memory. 
For example, a child reports that a boy rode a bicycle at the park at noon last Sunday. The child makes a judgement tracing back to the memory about how he or she acquired the information: Whether or not he or she actually witnessed the boy at the park, or has just heard the information (Johnson, Hashtoudi, \& Lindsay, 1993). This cognitive process includes simultaneous consideration of the information, while retaining it (i.e., a boy rode a bicycle at the park at noon last Sunday). This cognitive process fits the function of working memory (Gathercole \& Alloway, 2008). Recently, substantial attention has been paid to working memory as the rational intelligence of human beings (Alloway \& Alloway, 2013). The reliability of witness information (i.e., a boy rode a bicycle at the park at noon last Sunday) is dependent on source-monitoring ability, and if source-monitoring ability is closely related to working memory, we could estimate the reliability of witness information by examining the witness's working memory. Thus, in this paper, we examined the relationship between source-monitoring ability and working memory in young children. First, we review research on source monitoring among young children. Then, we discuss the relationship between source monitoring and working memory in young children.

Source-monitoring ability develops from infancy through childhood, and at approximately 10 years of age, children reach the adult-level of performance on a variety of source-monitoring tasks (Roberts, 2002). Johnson et al. (1993) classified source monitoring into three types: reality monitoring, monitoring of internal sources of information, and monitoring of external sources of information. Monitoring of external information sources refers to the distinction between external sources (e.g., identification of a speaker). Of these three types, young children develop the ability to monitor external information sources first, then that of reality monitoring, and finally, that of monitoring internal information sources (Kondo, 2012). For example, Foley, Johnson, and Raye (1983) showed that six-year-old children could identify two different speakers. Furthermore, the identification of two speakers is possible even for four-year-old children (Drummey \& Newcombe, 2002; Lindsay, Johnson \& Kwon, 1991). Monitoring of simple external sources of information is easy for young children.

However, young children have sometimes been shown to find the monitoring of external information sources difficult. In Kondo's (2008) study, 5- and 6-year-old participants heard words spoken by a man and a woman. Some words were spoken only by the man or the woman, and others by both or neither. The participants judged whether they heard words from a man, a woman, or both. It was difficult for the participants to monitor the sources of the words, especially those that they had heard from both the man and the woman. This result suggested that complex judgement about the memory source (i.e., A, B, or A and B) would require more cognitive resources, and that young children with limited cognitive resources would find such complex source-monitoring tasks difficult. This explanation is congruent with the idea that working memory is the basis for 
source monitoring. Therefore, next, we will discuss the relationship between source monitoring and working memory.

Working memory is the system responsible for temporarily storing and manipulating information (Gathercole \& Alloway, 2008). The model proposed by Baddeley and Hitch (1974), which postulated that this system consists of three limited-capacity elements (the central executive, phonological loop, and the visuospatial sketchpad), has been the basis of substantial research on working memory. The central executive is a domain-general component responsible for the control of attention and the processing involved in a range of regulatory functions, including the retrieval of information from long-term memory. In contrast, the phonological loop and the visuospatial sketchpad provide domain-specific temporary storage of verbal information, and of visual and spatial representations, respectively. We use "verbal working memory" to refer to the coordinated function of the phonological loop and the central executive, and "visuospatial working memory" to refer to the coordinated function of the visuospatial sketchpad and the central executive (Gathercole \& Alloway, 2008). A higher working memory capacity would enable children to use more cognitive resources and engage in source monitoring with ease. In addition, another working memory model by Cowan (2014) postulates that working memory is a part of an activated state of long-term memory, a smaller subset of which is attentional focus. According to this model, a higher working memory capacity would activate wider areas of long-term memory, which would leave more memory traces and render later source monitoring easier.

Recent research has shown that working memory is an important factor affecting source monitoring (e.g., Earhart \& Roberts, 2014; Gerrie \& Garry, 2007; Ruffman, Rustin, Garnham, \& Parkin, 2001). However, a few studies have examined the relationship between source monitoring and working memory among young children. Ruffman et al. (2001) exposed 6-, 8-, and 10-year-old participants to short stories on video or audiotape. The participants judged whether they had watched the story on video only, had listened to it on tape only, had both watched and listened to it, or had neither watched nor listened to the story. The participants also received a reading span test as a measure of their working memory ability. Findings revealed that the score on source monitoring was significantly related to working memory ability, suggesting that working memory ability should support young children's monitoring of external information sources. However, it was unclear whether working memory plays a similar role among preschool children.

Participants in Earhart and Roberts's (2014) study performed source-monitoring tasks of external information, working memory tasks (the WISC-IV digit span task), and tasks of an executive function of suppression. The participant groups were aged 4 - 6 years and 7 - 8 years. Working memory and suppression predicted source monitoring, but when the effect of age was controlled for, the influences of working memory and suppression on source monitoring disap- 
peared.

In summary, it is suggested that young children sometimes fail to monitor the source of external information because some seemingly do not develop enough working memory capacity. However, there has been no clear evidence concerning the direct relationship between working memory and monitoring of external information sources among young children. Moreover, working memory has two distinctive verbal and visual domains, but previous studies have focused only on verbal working memory (for example, by using the reading span and digit span tasks). Eyewitness testimony requires not only source monitoring for verbal information, but also visual information through words or pictures. Thus, it is important to include both verbal and visuospatial information in an experiment and examine the relationship between verbal and visuospatial working memories and source monitoring for the two types of information.

Therefore, in this study, we investigated how young preschool children's respective verbal and visuospatial working memory capacity would be related with source-monitoring tasks with verbally or visually presented stimuli. In this study, an adult man and an adult woman read picture books to children. Subsequently, the children were asked to judge the source of the verbal or visual materials and took working memory tests. We predicted that verbal working memory is positively correlated to the source monitoring of verbal stimuli, whereas visuospatial working memory is positively correlated to the source monitoring of visual stimuli.

\section{Method}

\subsection{Participants}

Four- ( $\mathrm{n}=21,10$ boys and 11 girls, mean age $=61.1$ months $)$ and five-year-old Japanese children ( $\mathrm{n}=21,10$ boys and 11 girls, mean age $=72.9$ months) participated in this study. Most participants belonged to middle-class families. Therefore, participants in this study were generally representative of children in Japan. The study was explained to the representatives of the participants' nursery school, and their consent was sought.

\subsection{Materials and Procedure}

As stimuli for the recognition and source-monitoring tasks that participants actually watched and listened to, we used two picture books written by the same author and illustrated by the same painter, namely "Teddy Bear Coalman" (Worthington \& Worthington, 1985/1987a) and "Teddy Bear Baker" (Worthington \& Worthington, 1985/1987b). We also used pictures and sentences from “Teddy Bear Postman" (Worthington \& Worthington, 1985/1987c) as false stimuli for the recognition source-monitoring tasks.

On the first day, participants watched and listened to the two picture books, and performed the recognition and source-monitoring tasks. First, an adult man and woman showed each picture book's pictures and read them out loud to 
groups of participants (each group comprised about five participants). The picture books consisted only of pictures, and there were no sentences. The storytelling sequence involving the two books, and the readers' genders were counterbalanced. Subsequently, experimenters different from the readers provided participants with the recognition and source-monitoring tasks individually. Stimuli of the tasks of the recognition and the source-monitoring tasks were voices of sentences and pictures, and the tasks were implemented in a block design with sentence voices or pictures as one block. The tasks of the block with sentence voices (the verbal recognition and the verbal source-monitoring tasks) or that with pictures (the visual recognition and the visual source-monitoring tasks) that were executed first differed for each participant. In the verbal recognition task, an experimenter verbally presented short sentences to a participant and asked, "Was this sentence presented or not presented? If the sentence was presented, please point to the circle (o) on this card. If the sentence was not presented, point to the cross $(x)$ on this card." After each verbal recognition task, the participants identified the source of the sentence, using photograph cards of the adult man and woman. The participants were asked whether they had heard the sentence from the book read by the adult man, woman, or neither. In the visual recognition task, the experimenter presented pictures to the participant and asked, "Was this picture presented or not presented? If the picture was presented, please point to the circle (o) on this card. If the picture was not presented, point to the cross $(x)$ on this card." After each recognition task, the participants identified the source of the picture, using photograph cards of the adult man and woman. The participants were asked whether they had seen the picture from the book read by the adult man, woman, or neither. The symbols " $\mathrm{o}$ " and " $x$ " are often used in a quiz to indicate "true" and "false," respectively, and young Japanese children are familiar with the symbols. Figure 1 shows the procedures of the recognition tasks and the source-monitoring tasks.

On the second and third days, the participants attempted four working memory tasks individually. Because it took about forty minutes to complete the

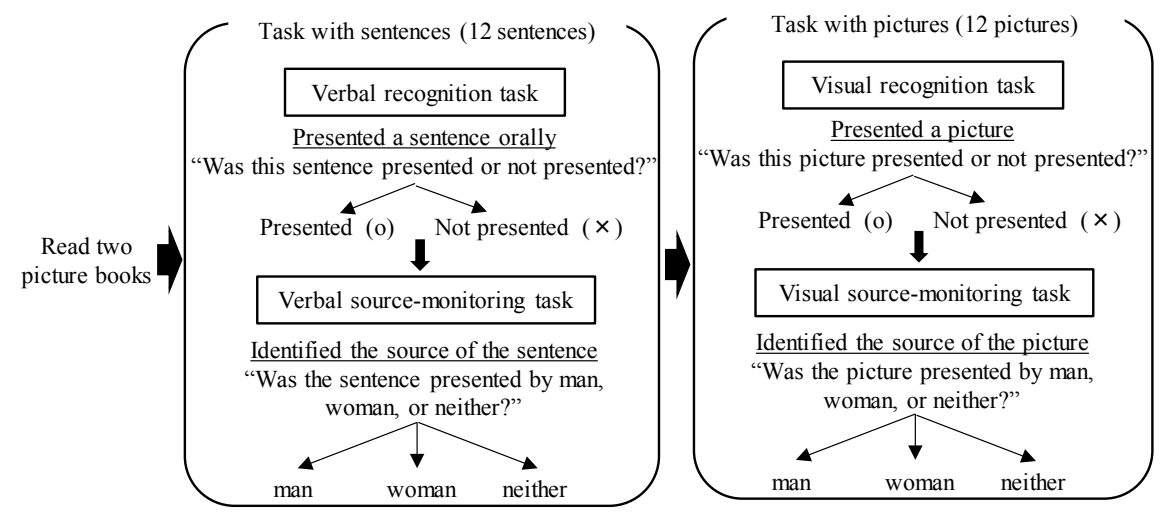

Figure 1. Procedure of the recognition and source-monitoring tasks. The task of the block with sentences or that with pictures that was executed first differed for each participant. 
four tasks, which seemed too long for young children, the participants engaged in a few tasks each day. In each working memory task, we provided children with practice of the task to confirm if the children understood the procedure. The order of the working memory tasks was counterbalanced.

\subsection{Recognition Tasks (Verbal Recognition Task and Visual Recognition Task)}

In the recognition task, 16 items were selected from each of the two picture books, "Teddy Bear Coalman" and "Teddy Bear Baker," as correct items, and eight were selected from the picture book "Teddy Bear Postman," as incorrect items. Half of the 24 items were presented in the verbal recognition task and the rest were presented in the visual recognition task. In the former, the items were presented verbally, using short sentences. For example, "Teddy bear read a picture book while sitting before a fireplace." Four of the verbal items from "Teddy Bear Coalman" and four from "Teddy Bear Baker" were presented to the same participants. Because the eight items were sentences that the participants had already heard, "o" was the correct response, and was regarded as a "hit." Four verbal items were also selected from "Teddy Bear Postman." Because these items were sentences that the participants had not heard, "o" was the incorrect response, and was regarded as a "false alarm" (FA). In the visual recognition task, the remaining 12 items were presented to participants by using pictures. The 12 items were presented verbally and the remaining 12 items were presented through pictures, to the same participants. The order of the presentation methods of the items was counterbalanced. We calculated the accuracy of recognition judgment $(d)$ by subtracting the FA rate from the Hit rate for each participant, divided by the standard deviation of the FA rate for each age group.

\subsection{Source-Monitoring Tasks (Verbal Source-Monitorng Task and Visual Source-Monitoring Task)}

After each recognition judgement, participants were asked about the source of the 12 verbal and 12 picture items in the recognition task. The participants judged whether the item was from the story book read by the adult man or from that read by the adult woman, or whether they had not seen or heard the story involving the item. The verbal source-monitoring task comprised judgements about the sources of the 12 short sentences, and the visual source-monitoring task comprised judgements about the sources of the 12 pictures. One point was awarded for each correct identification of the source for each of the 12 items. Thus, the maximum score on each source-monitoring task was 12 .

\subsection{Working Memory Tasks}

Participants completed two verbal working memory tasks, a backward digit span test, during which the participants heard a sequence of digits and had to repeat the sequence backwards, and a listening span test, in which they heard a series of 
sentences about animals and had to judge whether the sentences were correct, while remembering the animal that was first mentioned in the sentences. In the backward digit span task, participants expressed their answers aloud. In the listening span task, they judged the correctness of the sentences and named the first animal mentioned in the sentences aloud. The sequence of the digits and series of sentences increased one by one, starting from two and one, respectively. There were six trials for the same number of digit sequences and sentence series. Once participants succeeded in four of six trials for, for example, two-digit sequences, the six trials for three-digit sequences started. If participants failed in over four trials, tasks were discontinued.

Participants also completed two visuospatial working memory tasks, a comparative line test and a rotated figure test. In the visuospatial working memory tasks, they reported their answers by pointing to the response card. In the comparative line test, the participants were presented a pair of lines side by side, and they judged whether the lines were of the same length. The line on the right side was presented at a particular location in a lattice. The sequence of the presented pairs of lines increased by one, starting from one, and the participants had to remember the locations of the lines in the correct order. In the rotated figure test, the participants were presented with a pair of figures side by side, and they judged whether the direction of the figures was the same when one figure was rotated in their mind. The sequence of the presented pairs of figures increased by one, starting from one, and the participants had to correctly remember the sequence of the figures. There were six trials for the same number of sequences. When participants succeeded in four of six trials, for example, for two lines, the six trials for three lines were started. If participants failed in over four trials, the tasks were discontinued.

Participants acquired one point if they correctly remembered a digit sequence, an animal series, a sequence of the line locations, or a sequence of figures. For example, if a participant answered five two-digit sequences and two three-digit sequences correctly, the participant acquired 7 points.

\section{Results}

\subsection{Performance on the Working Memory, Recognition, and Source-Monitoring Tasks}

Table 1 shows participants' mean scores on the four tests related to the working memory task. The scores on the comparative line task were low irrespective of the participants' age. The comparative line task was so difficult for young children that it might be inappropriate for tapping the individual differences in their visuospatial working memory skills. Actually, the score on the comparative line task was not significantly correlated with that on the rotated figure task and age in months ( $r=.17$ and .17 , both ns., respectively). Therefore, the comparative line task was excluded from subsequent analyses.

Table 2 shows the $d$ 's and the percentages of participants' Hit and FA. 
Table 3 shows the participants' mean scores on the source-monitoring tasks.

Table 4 shows the correlations among the three tests of working memory (without the comparative line task), age in months, the recognition tasks, and the source-monitoring tasks. The correlations were generally moderate. The fact that most of the tasks in this study were positively correlated with each other seems to reflect the phenomenon referred to as, "positive manifolds" (Conway, Macnamara, \& Engel de Abreu, 2013). Moreover, the correlations between the three working memory tests were moderate, possibly because all these tests tapped the central executive construct suggested by Baddeley and Hitch (1974). However, considering the relatively small correlations between the three tests, the data relating to these working memory tests was handled separately for the subsequent analyses.

\subsection{Relationships of Working Memory with the Recognition Task and Source Monitoring}

To examine the effects of working memory on source monitoring, we conducted hierarchical multiple regression analyses using the scores on the verbal and visual recognition tasks, and the source-monitoring task as dependent variables. In the first step, age in months was entered as an independent variable, in order to control for the age effect. In the second step, we forcibly entered the scores on the three working memory tests.

Table 5 presents the results of the hierarchical multiple regression analyses with the scores on the verbal recognition and source-monitoring tasks. For the verbal recognition task, in the first step, when the variable "age in months" was entered, the model was nearly significant $(F(1,40)=3.48, p=.063)$. In the second step, the scores on the three tests of working memory (the backward digit span, listening span, and rotated figure) were entered forcibly, but these were not identified as significant predictors. On the other hand, for the verbal source-monitoring task, in the first step, when the variable "age in months" was entered, the model was significant $(F(1,40)=9.97, p=.003)$. In the second step, the scores on the three tests of working memory (the backward digit span, listening span, and rotated figure) were entered forcibly, and the "backward digit span" score emerged as a significant predictor. The explanatory power of the variable "age in months" was ultimately not significant.

Table 6 presents the results of the hierarchical multiple regression analyses with the scores on the visual recognition and source-monitoring tasks. For the visual recognition task, in the first step, when the variable "age in months" was entered, the model was significant $(F(1,40)=5.93, p=.020)$. In the second step, the scores on the three tests of working memory (the backward digit span, listening span, and rotated figure) were entered forcibly, and "listening span" was selected as a nearly significant predictor of the score on the visual recognition task. The explanatory power of the variable "age in months" was ultimately not significant. On the other hand, for the visual source-monitoring task, in the first 
Table 1. Ms, SDs, and ranges of scores on the four tests of the working memory tasks.

\begin{tabular}{ccccccc}
\hline & \multicolumn{3}{c}{ Four-year-olds } & \multicolumn{3}{c}{ Five-year-olds } \\
\hline & $M$ & $S D$ & Range & $M$ & $S D$ & Range \\
\hline Backward digit span & 5.0 & 3.9 & $0.0-13.0$ & 7.9 & 3.9 & $0.0-16.0$ \\
Listening span & 4.4 & 3.4 & $0.0-11.0$ & 6.9 & 3.2 & $0.0-14.0$ \\
Comparative line & 3.3 & 2.3 & $0.0-7.0$ & 4.1 & 2.4 & $1.0-11.0$ \\
Rotated figure & 5.6 & 2.9 & $0.0-11.0$ & 6.9 & 2.1 & $4.0-11.0$ \\
\hline
\end{tabular}

Table 2. $M$ s and $S D$ s of Hit (\%), FA (\%), and $d$ scores on the recognition task.

\begin{tabular}{cccccc}
\hline & & \multicolumn{2}{c}{ Four-year-olds } & \multicolumn{2}{c}{ Five-year-olds } \\
\cline { 2 - 6 } & & $M$ & $S D$ & $M$ & $S D$ \\
\hline & Hit & 83.9 & 18.2 & 85.7 & 16.0 \\
Verbal recognition task & FA & 42.9 & 35.5 & 31.0 & 36.1 \\
& $d$ & 1.2 & 1.0 & 1.5 & 1.1 \\
& Hit & 88.1 & 15.0 & 89.3 & 12.7 \\
Visual recognition task & FA & 20.2 & 31.2 & 13.1 & 25.8 \\
& $d$ & 2.2 & 1.1 & 3.0 & 1.0 \\
\hline
\end{tabular}

Table 3. $M s(S D s)$ of scores on the source-monitoring task.

\begin{tabular}{lcc}
\hline & Four-year-olds & Five-year-olds \\
\hline Verbal source-monitoring task & $6.4(1.9)$ & $7.7(2.5)$ \\
Visual source-monitoring task & $7.9(2.3)$ & $9.3(2.5)$ \\
\hline
\end{tabular}

Table 4. Correlations between the four tests of working memory, age in months, recognition tasks, and source-monitoring tasks.

\begin{tabular}{|c|c|c|c|c|}
\hline & $\begin{array}{l}\text { Backward } \\
\text { digit span }\end{array}$ & Listening span & Rotated figure & Age in months \\
\hline Listening span & $.33^{*}$ & & & \\
\hline Rotated figure & $.46^{\star *}$ & $.33^{*}$ & & \\
\hline Age in months & $.36^{*}$ & $.39^{*}$ & $.40^{* *}$ & \\
\hline Verbal recognition $(d)$ & $.27^{+}$ & $.30^{+}$ & $.37^{*}$ & $.29^{+}$ \\
\hline Verbal source-monitoring & $.52^{\star *}$ & $.45^{\star *}$ & $.41^{\star *}$ & $.45^{\star *}$ \\
\hline Visual recognition $(d)$ & $.32^{*}$ & $.42^{\star *}$ & $.37^{*}$ & $.36^{*}$ \\
\hline Visual source-monitoring & $.28^{+}$ & $.44^{\star *}$ & $.49^{\star *}$ & $.40^{\star *}$ \\
\hline
\end{tabular}


Table 5. Results of the hierarchical multiple regression analyses with verbal recognition and source-monitoring task scores $(\beta)$.

\begin{tabular}{ccccc}
\hline \multirow{2}{*}{ Explanatory variable } & \multicolumn{2}{c}{ Verbal recognition task $(d)$} & \multicolumn{2}{c}{ Verbal source-monitoring task } \\
\cline { 2 - 5 } & Step 1 & Step 2 & Step 1 & Step 2 \\
\hline Age in months & $.042^{+}$ & .015 & $.146^{* *}$ & .065 \\
Backward digit span & & .017 & & $.175^{\star}$ \\
Listening span & & .046 & .155 \\
Rotated figure & & .091 & .087 \\
$R^{2}$ & $.084^{+}$ & .184 & $.199^{* *}$ & $.400^{* *}$ \\
$\Delta R^{2}$ & .084 & .100 & .199 & .201 \\
\hline
\end{tabular}

${ }^{*} p<.01,{ }^{*} p<.05,{ }^{+} p<.10$.

Table 6. Results of the hierarchical multiple regression analyses with visual recognition and source-monitoring task scores $(\beta)$.

\begin{tabular}{ccccc}
\hline \multirow{2}{*}{ Explanatory variable } & \multicolumn{2}{c}{ Visual recognition task $(d)$} & \multicolumn{2}{c}{ Visual source-monitoring task } \\
\cline { 2 - 5 } & Step 1 & Step 2 & Step 1 & Step 2 \\
\hline Age in months & $.057^{*}$ & .023 & $.140^{* *}$ & .056 \\
Backward digit span & & .027 & & -.014 \\
Listening span & & $.088^{+}$ & $.188^{+}$ \\
Rotated figure & & .073 & $.328^{\star}$ \\
$R^{2}$ & $.129^{*}$ & $.264^{* *}$ & $.156^{* *}$ & $.342^{\star *}$ \\
$\Delta R^{2}$ & .129 & .135 & .156 & .186 \\
\hline
\end{tabular}

${ }^{* *} p<.01,{ }^{*} p<.05,{ }^{+} p<.10$.

step, when the variable "age in months" was entered, the model was significant $(F(1,40)=7.39, p=.010)$. In the second step, the scores on the three tests of working memory (the backward digit span, listening span, and rotated figure) were entered forcibly, and "rotated figure" was selected as a significant predictor of the score on the visual source-monitoring task. In addition, "listening span" was selected as a nearly significant predictor of the score on the visual recognition task. The explanatory power of the variable "age in months" was ultimately not significant.

\section{Discussion}

In this study, we investigated how young children's respective verbal and visuospatial working memory capacity would be related with their performance on source-monitoring tasks with verbal or visual stimuli. We predicted that verbal working memory is positively correlated to the source-monitoring of verbal stimuli, whereas visuospatial working memory is positively correlated to the source-monitoring of visual stimuli.

First, we discuss the effect of working memory on the performance of the 
recognition tasks. We found that the listening span test was a nearly significant predictor of performance on the visual recognition task ( $\beta=.088)$. However, it is unclear why this finding emerged. One possibility is that the participants used image strategies for the listening span task. In the listening span task, participants are required to remember the names of animals presented in each sentence. It was observed that, the present participants remembered animals' names with images instead of words. Therefore, this task may have been related visual recognition. Alternatively, the participants may have recognized the visual items in the picture books by name so that they could be located within the contexts of the story books. Another possibility is that these tests tap the same function of the central executive, which might have been related with the visual recognition task.

Second, and most importantly, we discuss the effect of working memory on the performance on the source-monitoring tasks. We found that the backward digit span significantly explained performance on the verbal source-monitoring task $(\beta=.175)$, whereas the rotated figure test significantly explained performance on the visual source-monitoring task $(\beta=.328)$. These results were exactly in keeping with what we had predicted. Young children with a higher verbal working memory capacity could judge the source of verbal items more accurately, whereas young children with a higher visuospatial working memory capacity could judge the source of visual items more accurately. Incidentally, the listening span task was a nearly significant predictor of the score on the visual source-monitoring task. This might be accounted for by the fact that working memory tests tap the same function of the central executive.

Why did the different working memory tests explain the verbal and visual recognition, and source-monitoring tasks? In the source-monitoring tasks, children were required to remember not only the contents of the picture books, but also the contexts in which the picture books were read to the children. Because young children tend to rely on visuospatial working memory than they do on other types of memory when watching and listening to an adult reading and showing picture books (Yuzawa, 2014), their memory representation for the events relating to picture books that they had seen might have been structured more in terms of a sequence of visual images, relatively independent of and loosely related to a sequence of verbal information. Therefore, in the visual source-monitoring task, they tended to rely on visuospatial working memory to remember and check visually presented information, whereas in the verbal source-monitoring task, they tended to rely on verbal working memory to remember and check verbally presented information.

Of the two tests of visuospatial working memory, only the "rotated figure" test explained performance on the visual source-monitoring task. This is because the stimuli in the visual recognition and source-monitoring tasks included visual objects, and they were related to visual information that had been incorporated into the "rotated figure" test. There is evidence for the fractionation of visuospa- 
tial working memory into visual and spatial subcomponents (Logie, 1986), and the "rotated figure" test weakly correlated with the "comparative line" test.

On the other hand, only the "backward digit span" test explained the performance on the verbal source-monitoring task. The score on the "listening span" test was lower than that on the "backward digit span" task, as seen in Table 1. It seems that the "listening span" task was difficult for participants, and therefore, it might not reflect their individual differences in verbal working memory capacity appropriately. In addition, as mentioned above, participants might have used the image strategy for the listening span task. Thus, the listening span task might not be an appropriate test for assessing verbal working memory.

In summary, children with a higher verbal working memory capacity obtained a higher score on the verbal source-monitoring task, whereas children with a higher visuospatial working memory capacity obtained a higher score on the visual source-monitoring task. These findings suggest that the effects of working memory on young children's monitoring of external information sources interacted with the domain of working memory and the format of source monitoring.

Finally, the findings of this study have an important implication for young children's eyewitness identification for investigating and prosecuting crimes. In order to increase the accuracy of eyewitness identification among young children, it is important that their working memory abilities be examined and that questions be asked in a format that the children are fairly competent in understanding.

\section{Acknowledgements}

This work was supported by JSPS KAKENHI Grant Numbers JP17J02824, JP17H02635.

\section{References}

Alloway, T. P., \& Alloway, R. G. (2013). Working Memory: Connected Intelligence. New York: Psychology Press.

Baddeley, A. D., \& Hitch, G. (1974). Working Memory. Psychology of Learning and Motivation, 8, 47-90. https://doi.org/10.1016/S0079-7421(08)60452-1

Conway, A. R. A., Macnamara, B. N., \& Engel de Abreu, P. M. J. (2013). Working Memory and Intelligence: An Overview. In T. P. Alloway \& R. G. Alloway (Eds.), Working Memory: Connected intelligence (pp. 13-36). New York: Psychology Press.

Cowan, N. (2014). Working Memory Underpins Cognitive Development, Learning, and Education. Educational Psychology Review, 26, 197-223. https://doi.org/10.1007/s10648-013-9246-y

Drummey, A. B., \& Newcombe, N. S. (2002). Developmental Changes in Source Memory. Developmental Science, 5, 502-513. https://doi.org/10.1111/1467-7687.00243

Earhart, B., \& Roberts, K. P. (2014). The Role of Executive Function in Children's Source Monitoring with Varying Retrieval Strategies. Frontiers in Psychology, 5, 1-12. 
https://doi.org/10.3389/fpsyg.2014.00405

Foley, M. A., Johnson, M. K., \& Raye, C. L. (1983). Age-Related Changes in Confusion between Memories for Thoughts and Memories for Speech. Child Development, 51-60. https://doi.org/10.2307/1129860

Gathercole, S. E., \& Alloway, T, P. (2008). Working Memory and Learning: A Practical Guide for Teachers. London, US: Sage.

Gerrie, M. P., \& Garry, M. (2007). Individual Differences in Working Memory Capacity Affect False Memories for Missing Aspects of Events. Memory, 15, 561-571. https://doi.org/10.1080/09658210701391634

Johnson, M. K., Hashtroudi, S., \& Lindsay, D. S. (1993). Source Monitoring. Psychological Bulletin, 114, 3-28. https://doi.org/10.1037/0033-2909.114.1.3

Kondo, A. (2008). Developmental Changes in the External Source Monitoring Ability of Young Children. Japanese Journal of Developmental Psychology, 19, 47-56.

Kondo, A. (2012). The Development of Source Monitoring. In M. Yuzawa, S. Sugimura, \& M. Kenichi (Eds.), Educational and Developmental Psychology (pp. 90-103). Kyoto: Mineruba-Publisher.

Lindsay, D. S., Johnson, M. K., \& Kwon, P. (1991). Developmental Changes in Memory Source Monitoring. Journal of Experimental Child Psychology, 52, 297-318. https://doi.org/10.1016/0022-0965(91)90065-Z

Logie, R. H. (1986). Visuo-Spatial Processing in Working Memory. The Quarterly Journal of Experimental Psychology, A: Human Experimental Psychology, 38, 229-247. https://doi.org/10.1080/14640748608401596

Roberts, K. P. (2002). Children's Ability to Distinguish between Memories from Multiple Sources: Implications for the Quality and Accuracy of Eyewitness Statements. Developmental Review, 22, 403-435. https://doi.org/10.1016/S0273-2297(02)00005-9

Ruffman, T., Rustin, C., Garnham, W., \& Parkin, A. J. (2001). Source Monitoring and False Memories in Children: Relation to Certainty and Executive Functioning. Journal of Experimental Child Psychology, 80, 95-111. https://doi.org/10.1006/jecp.2001.2632

Worthington, P., \& Worthington, S. (1987a). Teddy Bear Coalman (Ishii, M. Trans.). Tokyo: Fukuinkan-Publisher. (Original Work Published 1985)

Worthington, P., \& Worthington, S. (1987b). Teddy Bear Baker (Ishii, M. Trans.). Tokyo: Fukuinkan-Publisher. (Original Work Published 1985)

Worthington, P., \& Worthington, S. (1987c). Teddy Bear Postman (Ishii, M. Trans.). Tokyo: Fukuinkan-Publisher. (Original Work Published 1985)

Yuzawa, M. (2014). Working Memory and Learning of the Language. In M. Yuzawa, \& M. Yuzawa (Eds.), Working Memory and Education (pp. 99-115). Kyoto: Kitaohji-Publisher. 